\title{
Método de análisis biomecánico de la marcha en pacientes portadores de prótesis de rodilla
}

\author{
MORGENSTERN, R. \\ Director del Laboratorio de Biomecánica y Profesor de la Esculea de Medicina de la Educación Física y el Deporte, \\ Universidad de Barcelona. \\ PARREÑO CATALÁN, \\ Licenciando en Educación Física, \\ doctorando en la Universidad de Barcelona. \\ J.L.; RUANO GIL, D. \\ Catedrático de Anatomia Humana, Director y Pofesor de la Escuela de Medicina de la Educación Fisica y el Deporte, \\ Universidad de Barcelona.
}

\section{RESUMEN}

El análisis de la marcha ha adquirido en los últimos tiempos un extraordinario desarrollo. En la actualidad la ciencia biomecánica ofrece un gran campo de actuación para el estudio de la patología de la deambulación, aplicando los conocimientos anatómicos, las leyes físicas y la alta tecnología (ordenadores, video, plataformas de fuerza, etc...)

En este estudio, se comparan por medio del análisis del movimiento en tres dimensiones, la dinámica y el comportamiento articular de la marcha normal y de la marcha en el paciente portador de protesis de rodilla unilateral y bilateral, para analizar si se aprecian diferencias en las características generales de la marcha y los cambios evolutivos que provocan estas diferencias y sus posibles repercusiones patológicas.

La marcha normal tiene una fase de balanceo, seguida de una fase de apoyo con: choque de talón, apoyo del retropié, apoyo de la planta, rodadura sobre el borde extremo, despegue del talón, despegue del primer dedo.

Esta dinámica se acompaña de balanceo de las extremidades superiores para mantener el equilibrio y compensar los momentos de inercia provocados por la zancada.

\section{SUMMARY}

The analysis of the walk has become in the last times into an extraordinary development. Actually the biomechanic science offers a large field of action in the study of the pathology of walking, using the anathomical knowledges, physical laws and high technology(computers, video, strength platforms etc..).

In this study, we have compared by means of the movement analysis in three dimensions, the dynamic and joint behaviour of normal walk and walk of the pacient carrier of a knee protesis, both uni and bilateral. to determine if any differences in the general walk caracteristics are appreciated and the evolutive changes that they produce and the possible pathological consequences.

The normal walk has a balancing stage, followed by a support stage with : heel bump, support of the retrofoot, sole of the foot support, rotation over the external border, heel takeoff, takeoff of the first toe.

This dynamic is produced together with a balancing movement of the upper extremities in order maintain the balance and to compensate the inertia moments produced by the step.

En la figura 1 puede verse un modelo biomecánico representando en el parte superior (marcada 1) la marcha en un individuo normal y en la parte inferior (marcado 2) la marcha en un individuo anciano, portador de prótesis de rodilla.

La marcha en la tercera edad, sin ser necesariamente patológica, acorta la longitud del paso, disminuye la frecuencia y desaparece el balanceo de las extremidades superiores, entre otras variaciones.

En este estudio, se comparan por medio del análisis del movimiento en tres dimensiones, la dinámica y comportamiento articular de la marcha normal y de la marcha en pacientes portadores de prótesis de rodilla unilateral y bilateral, para analizar los cambios si los hay y sus posibles repercusiones patológicas.

\section{MATERIAL Y MÉTODOS}

Este trabajo se ha realizado con los equipos de análisis del movimiento del Laboratorio de Biomecánica de la Escuela de Medicina de la Educación Física y el Deporte de la Facultad de Medicina de la Universidad de Barcelona.

Este laboratorio dispone de un moderno equipo para el análisis del movimiento humano, especialmente aplicable al análisis de la marcha humana. 

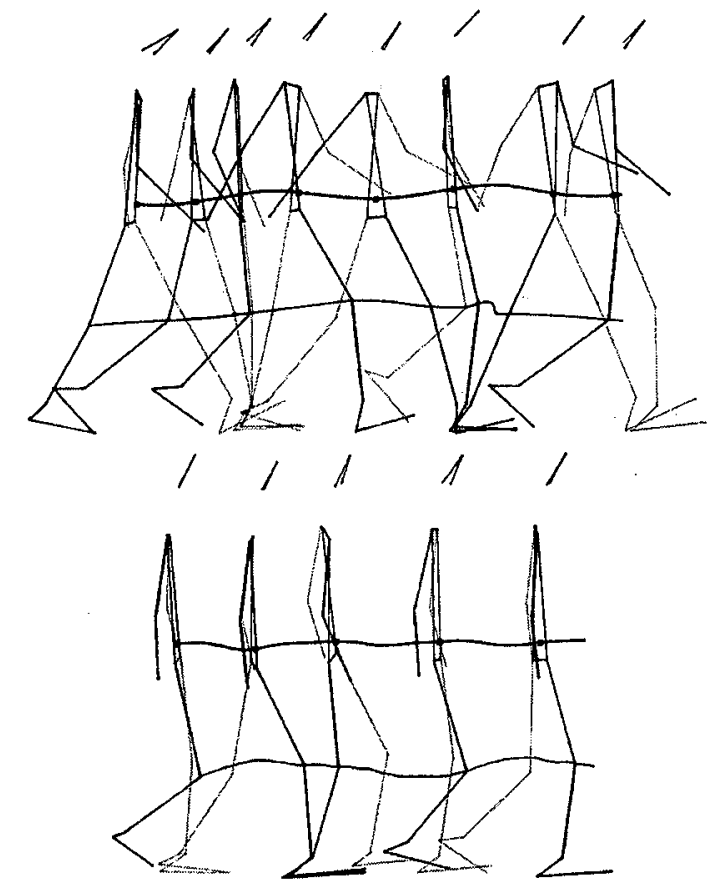

Figura 1.

Este equipo es capaz de representar un modelo biomecánico del sujeto en tres dimensiones, a partir de las imágenes de video de alta resolución obtenidas por tres ó más cámaras de filmatación. La filmación puede realizarse tanto en el exterior (pruebas de campo) como en el laboratorio de Biomecánica. La frecuencia de imágenes de la filmación en video es de 50 Herz.

La digitalización se realiza por medio de potentes programas de ordenador (Peak Performance, Colorado USA).

Se utiliza un marco de calibración para parametrizar el volumen espacial en el cual se realizará la toma de imágenes. Dicho marco proporciona los coeficientes del sistema de ecuaciones DLT que se utilizan para la parametrización espacial del modelo biomecánico.

Se pueden utilizar dos y tres cámaras de video de alta resolución para la filmación desde diversos ángulos, para conseguir la representación tridimensional.

La confección de un proyecto biomecánico comprende desde la elaboración de un modelo capaz de representar al sujeto ó a una parte del mismo en movimiento, hasta el marcado del sujeto y la correcta colocación de las cámaras.

Tras la filmación, se digitalizan los puntos del modelo sobre cada secuencia de película de video y se integra el conjunto en el ordenador para obtener una imagen tridimensional, en color, que representa al sujeto en movimiento real.
Esta imágen puede modificarse por segmentos y observarse desde cualquier perspectiva en el espacio, rotando alrededor de tres ejes ortogonales.

A partir del modelo de ordenador se pueden obtener los datos y las gráficas de la trayectoria, velocidad lineal y aceleración lineal de cada punto del modelo y el valor de los ángulos definidos, la velocidad angular y la aceleración angular, en función del tiempo de la secuencia digitalizada.

En la gráfica inferior se representan en ordenadas los grados de flexoextensión de la rodilla versus el tiempo de secuencia en abcisas. En trazo contínuo puede verse la evolución angular de la rodilla derecha y en trazo discontínuo la de la rodilla izquierda. Nótese la asimetría de los trazados.

De la misma filmación pueden obtenerse imágenes para realizar el análisis antropométrico del sujeto por medio de otro potente sistema informático, que permite el tratamientode imágenes y el cálculo de areas, perímetros, centroides, etc...

De los datos obtenidos, puede analizarse la biomecánica del sujeto y comparar a distintos individuos en diferentes ensayos.

\section{RESULTADOS Y DISCUSIÓN}

Se filmaron 34 ancianos, con edades comprendidas entre los 65 y los 80 años, 25 de sexo femenino y 9 de sexo masculino y se digitalizaron las imágenes de cada toma por ordenador. Se reconstruyó el movimiento por medio de un modelo biomecánico y se analizaron los parámetros siguientes:

- Longitud del paso

- Frecuencia del paso

- Evolución angular de cadera, rodilla y tobillo

- Trayectoria del centro de gravedad instantáneo

- Desplazamiento vertical y transversal del CDG.

En la figura 3 puede verse, en la parte superior, la evolución angular de la cadera derecha (trazo contínuo) e izquierda (trazo discontínuo) de un sujeto experimental versus el tiempo de la secuencia. En la parte inferior se representa la evolución angular de la rodilla derecha (trazo continuo) y de la rodilla izquierda (trazo discontínuo).

A título de ejemplo, en la figura 4 se ha representado en ordenadas la distancia longitudinal recorrida en metro y en abcisas el tiempo de la secuencia. El recorrido de un punto del talón derecho se ha representado en trazo continuo y el de el talón izquierdo en trazo discontínuo.

Cuando cualquiera de los dos trazos es paralelo al eje de abcisas, ello significa que no varía su coordenada en el sentido de la marcha, ó sea el talón en cuestión esta quieto sobre el suelo.

Por lo tanto la diferencia entre los trazos horizontales de ambos talones, medidos sobre el eje de ordenadas, dará la longitud del paso del individuo. 


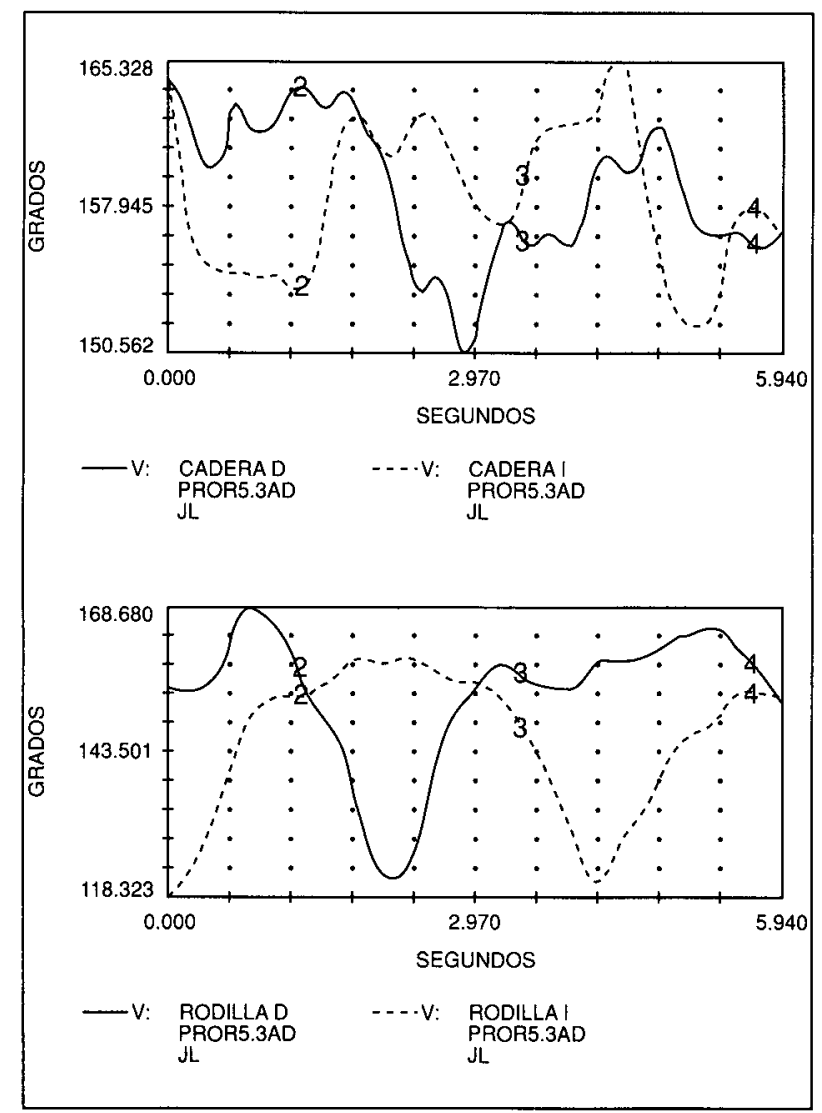

Figura 3.

Figura 1.

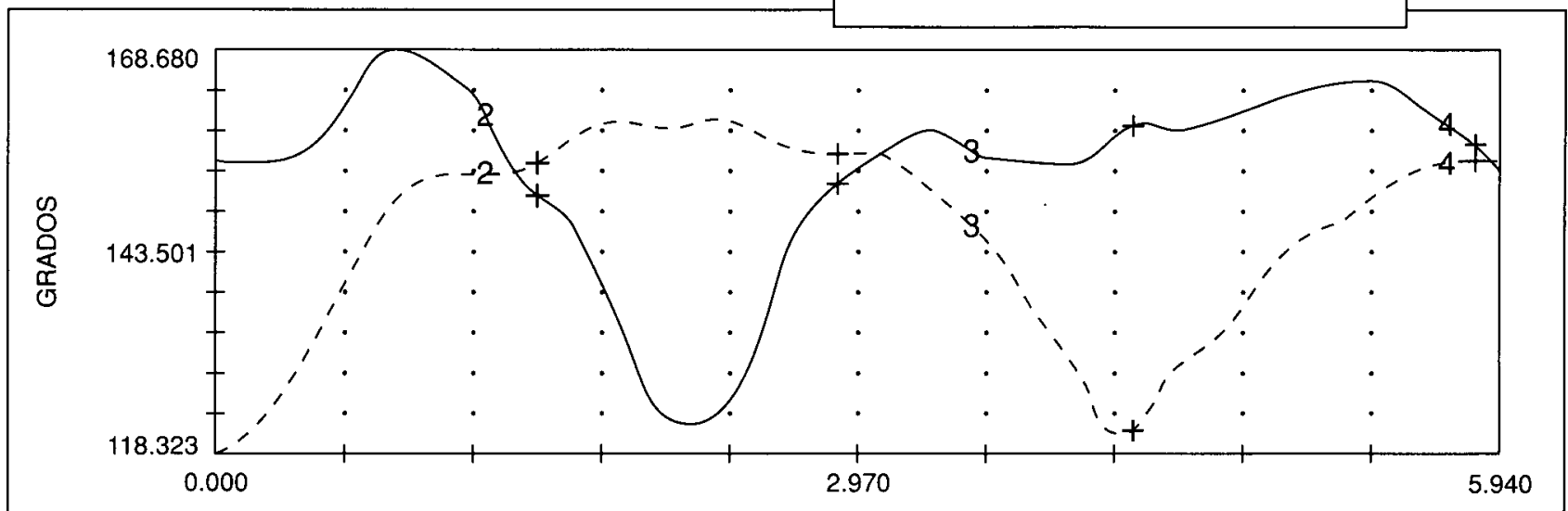


TABLA 1

TABLA DE MOVILIDAD ARTICULAR

\begin{tabular}{lccccc}
\hline & Tipo & P. Bil & P. Uni & Uni. D & Unil \\
\hline Cadera D & 44 & 25 & 25 & 27 & 23 \\
Cadera I & 44 & 23 & 22 & 23 & 20 \\
Rodilla D & 60 & 42 & 42 & 47 & 37 \\
Rodilla I & 60 & 40 & 39 & 43 & 35 \\
Tobillo D & 21 & 22 & 22 & 22 & 22 \\
Tobillo I & 21 & 19 & 21 & 21 & 22 \\
\hline
\end{tabular}

TABLA 2

TABLA DE RESULTADOS

\begin{tabular}{lccccc}
\hline & $\begin{array}{c}\text { Velocidad } \\
\text { Media } \\
\mathrm{m} / \mathrm{seg}\end{array}$ & $\begin{array}{c}\text { Desviación } \\
\mathrm{mm}\end{array}$ & $\begin{array}{c}Y \\
\mathrm{~mm}\end{array}$ & $\begin{array}{c}\text { Long. } \\
\text { paso } \\
\mathrm{cm}\end{array}$ & $\begin{array}{c}\text { Frec. } \\
\text { paso } \\
\text { paso/seg }\end{array}$ \\
\hline Control & 1.14 & 40 & 40 & 80 & 2 \\
P. Bilat. & 0.51 & 80 & 24 & 31 & $<1$ \\
P. Unil. & 0.56 & 74 & 23 & 24 & $<1$ \\
\hline
\end{tabular}

De la misma forma, la diferencia entre el inicio de cada escalón, medido sobre el eje de abcisas, dará el tiempo del paso ó sea permitirá el cálculo de la frecuencia.

El análisis del modelo biomecánico y en especial de la evolución angular durante el ciclo de la marcha de los sujetos, reveló los resultados expuestos en la tabla 1 .

En la primera columna se representan los valores normales (en grados) de amplitud articular durante la marcha. En las columnas siguientes se representan los valores medios de amplitud articular de los pacientes portadores de prótesis bilateral, prótesis unilateral derecha y prótesis unilateral izquierda.

Los valores angulares de rodilla y cadera disminuyen drásticamente respecto a la movilidad normal, indicando una menor movilidad articular en rodilla y cadera, sin embargo no hay diferencia entre la marcha normal y la protésica en el valor angular del tobillo.

En las prótesis unilaterales no se ha podido establecer una correlación entre los valores de movilidad articular de la rodilla protésica y de la no afecta, no siendo obvio que la rodilla prótésica tenga una disminución de la movilidad articular durante la marcha, estadisticamente.

En la tabla 2 se presentan los resultados de los parámetros cinemáticos como velocidad media del centro de gravedad, desplazamiento horizontal y vertical del centro de gravedad en el plano frontal, longitud del paso y frecuencia del paso.

En la primera fila se han representado los valores normales de control de un individuo. En la segunda fila los correspondientes a portadores de prótesis bilateral y en la tercera a portadores de prótesis unilateral.

La frecuencia y la longitud del paso disminuyen en todos los individuos, pero sobretodo en los que presentaban un postoperatorio más reciente.

La trayectoria del centro de gravedad instatáneo se aplana desapareciendo la trayectoria sinusoidal que se aprecia en el control. Ver figura 5 (arriba normal, abajo protéstico). Esto se traduce en una disminución del valor de desviación vertical y del centro de gravedad respecto al valor normal.

En la figura 6 puede verse a vista de pájaro la trayectoria del centro de gravedad, arriba en el protésico y abajo en el individuo normal. En el protésico aumenta la desviación horizontal $\mathrm{X}$ del centro de gravedad respecto al valor normal.

Desaparece casi por completo el balanceo de extremidades superiores.

Aparece en algún caso la presencia de patología articular, como el genu valgo ó el genu flexo, como mal resultado operatorio.

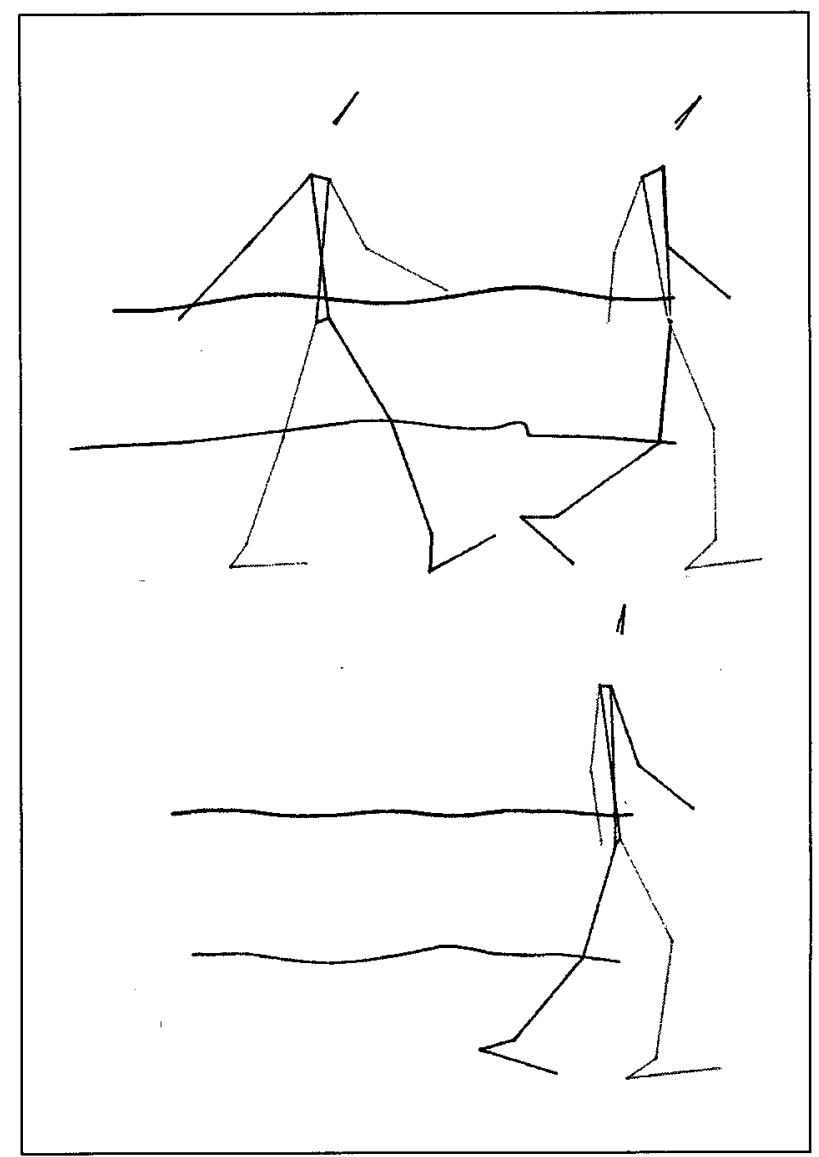

Figura 5. 
En conclusión se puede afirmar que la marcha en el paciente protésico se vuelve menos «móvil», probablemente para minimizar el gasto energético por una parte (aplanamietno de la trayectoria del CDG y menos basculación de las extremidades superiores, menor frecuencia de marcha) y por otra parte para disminuir al máximo la amplitud articular (zancada mas corta, menores ángulos de excursión articular) para evitar sobrecargas y dolor articular tras el ejercicio.

La inestabilidad de la marcha aumenta, con desviaciones sobre todo en el plano transversal, (incremento de $\operatorname{las} \mathrm{X}$ ).

No se ha podido establecer un aumento ó disminución de la movilidad articular en la rodilla protésica unilateral, comportandose estadísticamente de forma similar a la rodilla no intervenida.

\section{BIBLIOGRAFÍA}

- DEMOTTAZ, J.D.; MAZUR, J.M.; THOMAS, W.H.; SLEDGE, C.B. and SIMON, S.R.: Clinical Study of Total Ankle Replacement with Gait Analysis. J. Bone and Joint Surg., 61-A:976-988, Oct 1979.

- ISACSON, J; BROSTROM, L.A.: Gait in Rheumatoid Arthritis: An Electrogoniometer Investigacion. J. Biomechanics. 21(6):451-457, 1988

- LEE KIRBY, R.; MARLOW, R.W.; MACLEOD, D.A.;: and MARBLE, A.E.: The Effect of Locomotion Speed on the Anterior Tibial Intramuscular Pressure of Normal Humans. J. Biomechanics. 21(5):357-360, 1988.

- McFADYEN, B.J., and WINTER, DA.A.: An Integrated Biomechanical Analysis of Normal Stair Ascent and Descent.J. Biomechanics. 21(9):733-744, 1988.

- PANDY, M.G.; and BERME,N.: Quantitative Assessment of Gait Determinants During Single Stance Via a Three-Dimensional ModelPart 2. Pathological Gait. J. Biomechanics 22 (6-7):725-733, 1988.

- PATRIARCO. A.G.; MANN R.W.; SIMON S.R. AND MAN-

SOUR J.M.: An Evaluation of the Approaches of Optimization Models in the Prediction of Muscle Forces During Human Gait. J. Biomechanics $14(8): 513-525,1981$.

- STOKES. V.P.; ANDERSSON C. and FORSSBERG H.: Rotational and Transtional Movement Features of the Pelvis and Thorax During Adult Human Locomotion. J: Biomechanics 22 (1):4350, 1989.

- STOKES V.P.; A Method for Obtaining the 3D Kinematics of the Pelvis and Thorax During Locomotion. Human Movement Science 3, 77-94, 1984.

- WHITE S.C.; YACK H.J. and WINTER D.A.: A ThreeDimensional Musculoskeletal Model for Gait Analysis. Anatomical Variability Estimates. J. Biomechanics $22(8), 885-893,1989$.

- WINTER D.A.; SIENKO S.E.: Biomachanics of Below-Knee Amputee Gait. J. Biomechanics 21 (5), 361-367, 1988.

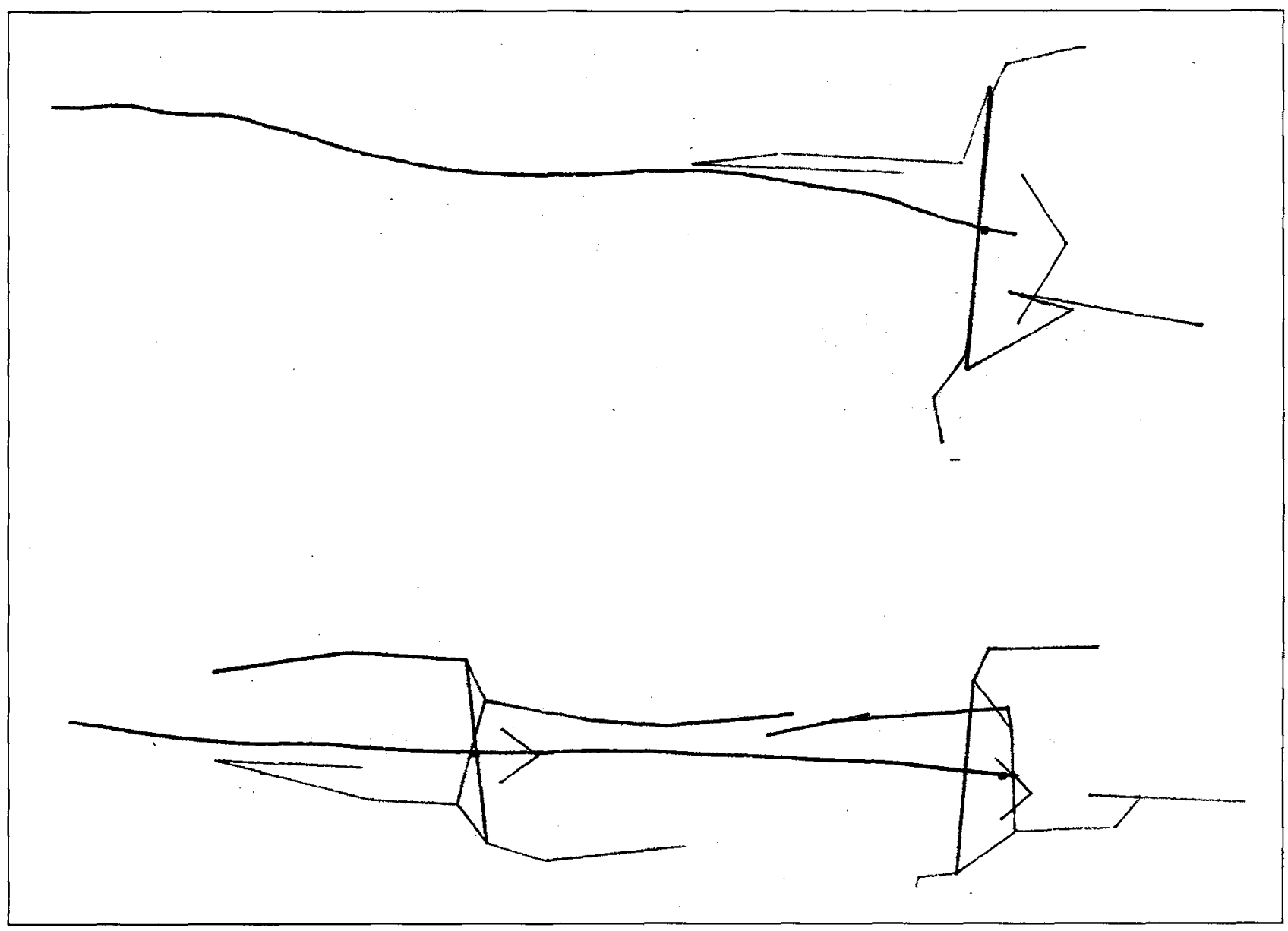

Figura 6. 